\title{
GESTIÓN DEL TALENTO HUMANO POR COMPETENCIAS PARA EL DESARROLlO DE CAPACIDADES DINÁMICAS, UN ACERCAMIENTO PARA EL DISEÑO DE SISTEMAS DE GESTIÓN
}

\author{
Management of human talent by competences for the development of dynamic \\ capacities, an approach for the design of management systems
}

\author{
Gisella Vizcaíno Solano ${ }^{1}$, Lucila Martes Rodríguez ${ }^{2}$, Winston Fontalvo Cerpa ${ }^{3}$, Ricardo Simancas Trujillo ${ }^{4}$
}

Recibido: Febrero 9 de 2016/Aceptado: Junio 22 de 2016

\begin{abstract}
RESUMEN
La gestión del talento humano es una función de vital importancia para las empresas, siendo un factor determinante en el logro de los objetivos corporativos. El presente artículo pretende revisar la teoría relacionada con la gestión del talento humano, los modelos de competencias y las capacidades dinámicas, con el propósito de establecer un marco que permita diseñar un modelo de gestión del talento humano por competencias para el desarrollo de estas últimas. Para ello presenta el abordaje teórico relacionado con la gestión del talento humano y las funciones de esta área en una organización, posteriormente analiza los modelos de gestión del talento humano por competencias, acercándose principalmente al propuesto por Martha Alles, por lo que se ahonda en este modelo; finalmente, se abordan las teorías relacionadas con capacidades dinámicas como fin de mejoramiento organizacional desde la gestión del talento humano. Se concluye que el diseño de este modelo le permite a la organización que lo implemente mayor competitividad, potenciado desde la gestión del talento humano. Se recomienda, a su vez, el compromiso decidido de todos, empezando por la alta dirección, así como crear un diccionario de competencias coherente con los propósitos estratégicos de la organización.
\end{abstract}

Palabras clave: Talento humano, Competencias, Capacidades dinámicas, Gestión del talento humano, Gestión por competencias.

\begin{abstract}
The management of human talent is a vital function for companies, being a determining factor in the achievement of corporate objectives. This article aims to review the theory related to human talent management, competency models and dynamic capabilities, in order to establish a framework that allows the design of a human talent management model by competencies for the development of dynamic capabilities. For this, it presents the theoretical approach related to the management of human talent and the functions of this area in an organization, later analyzes the models of the human talent management by competencies, has approached mainly to Martha Alles, reason why it is deepened in this model; Finally, they approach theories related to dynamic capabilities as an end to organizational improvement from the management of human talent. It concludes that the design of this model allows the organization that implements it greater competitiveness, enhanced from the management of human talent. Once again, the commitment of all is recommended, starting with senior management, as well as creating a dictionary of competencies that are coherent with the strategic purposes of the organization.
\end{abstract}

Keywords: Human talent, Competency, Dynamic capabilities, Management of human talent, Competency management.

Cómo referenciar este artículo: Vizcaíno, G., Martes, L., Fontalvo, W. \& Simancas, R. (2016). Gestión del talento humano por competencias para el desarrollo de capacidades dinámicas, un acercamiento para el diseño de sistemas de gestión. Ad-Gnosis, 5(5), 13-35.

1. Psicóloga. Magíster en Gerencia del Talento Humano. Profesora de tiempo completo, Corporación Universitaria Empresarial de Salamanca.g_vizcaino137@cues.edu.co

2. Psicóloga. Magíster en Gerencia del Talento Humano. Psicóloga orientadora, Combarranquilla.

martes lucila@hotmail.com

3. Ingeniero Industrial. Magíster en Educación. Docente investigador, Universidad Libre, seccional Barranquilla. winston.fontalvoc@unilibre.edu.co

4. Licenciado en Educación. Publicista. Magíster en Gerencia de Mercadeo. Docente investigador, Corporación Universitaria Americana. risitru@hotmail.com 


\section{Introducción}

Las organizaciones han implementado una serie de cambios que han permitido una evolución con el transcurrir del tiempo en todos sus procesos, especialmente en el área de gestión humana, hasta posicionar a las personas dentro de las organizaciones como un factor determinante en el logro de los objetivos de la compañía. Esto nos lleva a determinar que el éxito que puede lograr una organización depende de las personas, no de los procesos, por si solos, y al final los individuos se convierten en ese sello diferenciador entre una organización y otra.

Atendiendo a la necesidad de transformar el área de gestión humana, para convertirla en socio estratégico de la organización y responde a las exigencias del entorno competitivo, el área de gestión humana tiene un gran reto, pues a través de su labor día a día debe alcanzar los objetivos estratégicos. Eso significa promover una gestión del talento humano, que se interese no solo por competencias netamente técnicas, sino también competencias que permitan planificar estrategias de crecimiento y que ayuden al logro de los objetivos institucionales, por lo que resulta importante implementar un sistema de gestión por competencia que pueda dar aportes inmediatos, a las necesidades estratégicas del negocio (González, 2012).

Según la consultora Martha Alles (2013), "para trabajar con un sistema de gestión por competencias, es necesario definir, la visión, la mi- sión y los objetivos organizacionales y a partir de la dirección de la empresa, decidir cómo se hace".

\section{Gestión del talento humano}

En el área de la administración de empresas se habla de procesos administrativos, estrategias, objetivos organizacionales, recursos entre otros tantos términos que describen el mundo de las organizaciones. Y cada uno de estos implica un proceso y trabajo de un equipo interdisciplinario que se interesa por mantener en pie las organizaciones.

Históricamente la administración organizacional ha evolucionado como todo en la historia y ha pasado por diferentes modelos administrativos y cada uno de ellos explican cómo hacer uso de los recursos con los que cuenta la compañía, entre esos, nos encontramos con el recurso humano, el cual fue subvalorado durante mucho tiempo, pero gracias a movimientos sindicales y pronunciamientos de instituciones como la OIT y la ONU empieza a tomar importancia para la administración. Además del impacto que género en las organizaciones dos fenómenos en la administración que se dieron y son la generación de conocimiento para mejorar los procesos organizacionales y la teoría de la generación de ventaja competitiva a través del recurso humano (Valencia, Gutiérrez \& Rubio, 2013).

Las organizaciones dependen directa e irremediablemente de las personas, para operar, producir bienes y servicios, atender a los clientes, 
competir en los mercados y alcanzar objetivos generales y estratégicos. Esto ha dirigido la administración a comprometerse aún más con sus colaboradores, porque es seguro que las organizaciones jamás existirían sin estos que les dan vida, dinámica, impulso, creatividad y racionalidad (Chiavenato, 2013).

El área de gestión del talento humano es muy sensible a los cambios que se presentan en su entorno. Es contingente y situacional, pues depende de aspectos como la cultura, la estructura adoptada, la tecnología utilizada, los procesos internos, entre otras variables y todas ellas dependen de las personas (Chiavenato, 2013). Gestionar el talento humano de una organización implica integrar de todas las áreas de la empresa con el desempeño, la cultura y el clima de las personas que en ella trabajan.

La gestión del talento humano es el proceso de contratar, capacitar, evaluar, remunerar a los colaboradores de la organización, así como atender los aspectos de las relaciones laborales, su desarrollo personal y profesional, además de vigilar por la salud y seguridad laboral y aspectos legales (Rodríguez \& Sabastián, 2014).

Como los trabajadores son considerados en la actualidad un socio estratégico de la organización, esta debe vincularlos a la estrategia de la misma, ya que son proveedoras de conocimientos, habilidades, capacidades indispensables y la inteligencia, que permite tomar decisiones racionales e imprime significado y rumbo a los objetivos generales, generándole a la organización capital intelectual, considerado como ventaja competitiva para las organizaciones (Chiavenato, 2013). Esto implica valorar a los trabajadores pues son los que permitirán alcanzar los objetivos de la empresa, y la gestión humana se convierte en el puente de conexión entre la administración y el desempeño de los colaboradores (Chiavenato, 2013; Pardo \& Porras, 2011; Dessler, 2009).

Es importante resaltar que las actividades del área de talento humano son las que garantizan tener personal competente, por tal motivo se debe desarrollar todo tipo de competencias que posean los empleados para aumentar el capital humano en la organización (Chiavenato, 2011).

\section{Funciones de talento humano}

El éxito del trabajo del área de gestión humana está determinado por la manera de cómo esta desarrolla las diferentes actividades, las cuales son denominadas prácticas de gestión humana y que se describen a continuación.

1. Análisis de puestos. Para realizar el análisis del puesto se requiere de entrevistas con expertos en la materia y el acompañamiento de la persona encargada del área de gestión humana y se puede hacer uso de la observación, que da información de primera mano para describir con precisión los procesos (Rodríguez \& Sabastián, 2014).

2. Reclutamiento del personal. Es el proceso de divulgar información sobre vacantes, y obtener un grupo de candidatos cualifica- 
dos para un puesto específico, este puede hacerse a nivel interno o externo. Este proceso depende en gran medida de las necesidades de la empresa y de la dinámica de oferta y demanda de la mano de obra, tendencias del mercado y las exigencias de la aldea global (Chiavenato, 2011).

3. Selección del personal. Proceso que busca entre los candidatos reclutados el candidato que más se ajuste a las exigencias del puesto. El objetivo de la selección es escoger los candidatos idóneos para el cargo, seleccionados los mejores candidatos, se procede a la aplicación de pruebas que consiste en que ellos desarrollen una serie de pruebas de conocimiento, actitudinales, inteligencia, psicológicas y de personalidad, entre otras, aunque estas varían, dependiendo del cargo para el cual se aplica, también se desarrolla una entrevista con los candidatos al cargo y se procede a la verificación de referencias y de la información de la hoja de vida, en ocasiones es necesario realizar visitas domiciliarias y finalmente si este es contratado se le hace el proceso de contratación e inducción (Chiavenato, 2011).

4. Sistema de compensación. Son los procesos utilizados para incentivar a las personas y satisfacer sus necesidades individuales más sentidas. Este aspecto incluye los procesos de remuneración; programas de incentivos; y beneficios y servicios. Son recompensas cuantificables que recibe un empleado por su trabajo (Parker, McAdams \& Zielinski, 2013). La compensación ofreci- da por la organización influye en la satisfacción de los trabajadores, ya que en la medida en que sean mejor recompensados, su esfuerzo y empeño serán más visibles (Chiavenato, 2011).

Los elementos que componen la remuneración son: 1) Remuneración básica, que se otorga por horas o por mensualidades; 2) Incentivos salariales, son premios por el buen desempeño de los trabajadores; y 3) Otros beneficios que son todas aquellas recompensas representadas en (vacaciones, seguro de vida, seguro de salud, restaurante o comedor subsidiado, transporte, etc.) (Chiavenato, 2011).

\section{Capacitación y desarrollo}

La capacitación es un programa de la empresa que busca a través de la formación, desarrollar o fortalecer habilidades para que estos puedan estar mejor preparados y tener un mejor desempeño. A través de la capacitación la gerencia puede alcanzar la estrategia, ya que se pueden desarrollar las habilidades y competencias necesarias para lograr los objetivos establecidos en el plan estratégico. Por ello los planes de capacitación de la organización tienen que estar alineados a la estrategia de la misma (Castro, Miguel, Elizabeth \& Sánchez, 2013).

También hace parte del programa de capacitación y desarrollo el plan de carrera que consiste en una sucesión de cargos ocupados por una persona, cargos cada vez más complejos y requieren de preparación y capacitación (Dessler, 2009; Chiavenato, 2011). 


\section{Evaluación del desempeño}

Abarca los procesos empleados para acompañar y controlar las diferentes actividades de los trabajadores y verificar resultados, con el fin de mejorar el desempeño de estos. Este aspecto incluye los programas de evaluación y los métodos de evaluación del desempeño de los individuos (Aguilera, 2010). Existen varios métodos para hacer una evaluación de desempeño, entre ellos están:

Evaluación de $360^{\circ}$. En esta actividad participan todos los actores involucrados en la vida laboral del empleado (jefe, compañeros, clientes y proveedores), es una evaluación completa ya que se recoge información del desempeño del empleado de todas las fuentes.

Evaluación ascendente. En esta el jefe es evaluado por sus subordinados para determinar la eficiencia con la que este suministró los recursos y logro las metas u objetivos.

Escalas gráficas. Es un método basado en una tabla de doble entrada, en que las filas muestran los parámetros básicos de evaluación y las columnas indican los grados de evaluación de desempeño.

Evaluación por selección forzada. Se evalúa el desempeño a través de unas frases que describen el comportamiento del trabajador y se deben escoger en pares y el evaluador debe escoger entre las frases que ya están predeterminadas.
Listas de verificación. Es una especie de recordatorio para que el gerente evalúe las características principales de un empleado (Chiavenato, 2011).

\section{Seguridad y salud laboral}

Esta hace referencia a los procesos utilizados para crear y mantener las condiciones ambientales y psicológicas satisfactorias para las actividades de las personas. Abarca los procesos de higiene, seguridad industrial y calidad de vida, las relaciones laborales y la ruptura laboral o finalización de contrato (Aguilera, 2010). Actualmente las aseguradoras de riesgos profesionales están acompañando los procesos relacionados con la salud y la seguridad industrial y se han convertido en un apoyo para el área de gestión humano, esta tiene la responsabilidad de ser un punto de apoyo en la construcción y mantenimiento de una cultura y clima laboral acorde a los lineamientos institucionales (Gelabert, 2014).

Los avances tecnológicos y el desarrollo del aprendizaje corporativo han logrado influir significativamente en los procesos organizacionales, estos cambios se han dado gracias a la participación activa de los empleados en procesos de desarrollo, lo cual ha obligado en gran medida a que la gestión humana se preocupe por mejorar el desempeño de los trabajadores a través del desarrollo y fortalecimiento de sus competencias, proponiendo mejores prácticas de su área (Moreno, 2012). 


\section{Competencia}

Etimológicamente, el origen de la palabra competencia en la lengua española se relaciona con los verbos competer y competir provienen de un mismo verbo latino: competere, que significa ir de una cosa al encuentro de otra, encontrarse, coincidir. A partir del siglo XV competer adquiere el significado de "pertenece a", incumbir, que da lugar al sustantivo competencia y al adjetivo competente, que indica apto o adecuado. También, el término competer en aquella época es usado como pugnar con, rivaliza con, contender, lo cual da origen a los sustantivos competición, competencia, competidor, competitividad y al adjetivo competitivo (Muñoz \& Vargas, 2016).

El concepto de competencia aparece ante la necesidad de realizar predicciones acerca de la ejecución de las instrucciones o tareas de la persona en su desempeño laboral (Beltrán \& Urrea, 2013; McClelland, 1973) y es utilizado con fuerza a partir de los años 80 , siempre asociado a las características psicológicas que posibilitan un desempeño superior. Para McClelland (1973) las competencias aparecen vinculadas a una forma de evaluar aquello que "realmente causa un rendimiento superior en el trabajo" y no "a la evaluación de factores que describen confiablemente todas las características de una persona, con la esperanza de que algunas de ellas estén asociadas con el rendimiento en el trabajo".

Es con esta relación que los siguientes autores definen la competencia:
Conjunto de características de una persona que están relacionadas directamente con una buena ejecución en una determinada tarea o puesto de trabajo. (Boyatzis 1982, citado por González, 2002)

Una característica subyacente de un individuo que está causalmente relacionada con un rendimiento efectivo o superior en una situación o trabajo. (Spencer \& Spencer, 1993, citado por González, 2002)

Conjunto de conocimientos, habilidades, disposiciones y conductas que posee una persona que le permiten la realización exitosa de una actividad. (Feliú \& Rodríguez, 1996, citado por González, 2002)

Una habilidad o atributo personal de la conducta de un sujeto que puede definirse como característica de su comportamiento y bajo la cual el comportamiento orientado a la tarea puede clasificarse de forma lógica y fiable. (Gonczi \& Athanasou, 1996 citado por Trujillo, 2014)

Si bien, se tienen muchas definiciones sobre las competencias, el enfoque que tiene el presente trabajo está más cercano a la definición de Gonczi y Athanasou, por cuanto su apreciación de competencia lleva la misma orientación que el trabajo de Martha Alles, tratado posteriormente. 
1. Las competencias son repertorios de comportamientos que algunas personas dominan mejor que otras, lo que las hace eficaces en una situación determinada (Ferreyra, 2014). Según este autor, son además observables en la realidad del trabajo, e igualmente en situaciones de test, y ponen en práctica de manera integrada aptitudes, rasgos de personalidad y conocimientos. Por lo tanto, las considera un trazo de unión entre las características individuales y las cualidades requeridas para llevar a cabo las misiones del puesto.

2. Las competencias son unas características subyacentes a la persona, que están casualmente relacionadas con una actuación exitosa en el puesto de trabajo (Boyatzis, 1982 citado por Torres, 2015).

3. Se refiere a la capacidad efectiva para llevar a cabo exitosamente una actividad laboral plenamente identificada (según la OIT este es el concepto generalmente más aceptado).

4. Es la idoneidad para realizar una tarea o desempeñar un puesto de trabajo eficazmente, por poseer las calificaciones requeridas para ello (OIT).

De una forma u otra, puede concluirse que, de manera general, están contenidos los cincos tipos de características competenciales propuestas por Lyle M. Spencer y Signe M. Spencer citados por Ruiz y Rodríguez (2012):

Para Spencer y Spencer los principales tipos de competencias son los siguientes (Alles, 2006):
1. Motivación: Los intereses que considera o desea una persona consistentemente. Las motivaciones "dirigen, conllevan y seleccionan" el comportamiento hacia ciertas acciones o lo alejan de otros. Ejemplo: Las personas motivadas que desean éxito se establecen constantemente objetivos, toman responsabilidad para lograr metas.

2. Características: Rasgos físicos y respuestas consistentes a situaciones o información. Ejemplo: Tiempo de reacción y buena vista, son competencias físicas de los pilotos de combate. El autocontrol y la iniciativa son "respuestas consistentes a situaciones" más complejas, algunas personas no "molestan" a otras y actúan "por encima y más allá del llamado del deber" para resolver problemas bajo estrés.

3. Concepto propio o autoconcepto: Las actitudes, valores o imagen de una propia persona. Ejemplo: La confianza de poder desempeñarse bien en casi cualquier situación.

Spencer y Spencer introducen el "Modelo de Iceberg" donde gráficamente dividen las competencias en dos grandes grupos: las más fáciles de detectar y desarrollar, como las destrezas y conocimientos y las menos fáciles de detectar y de desarrollar, como el concepto de uno mismo, las actitudes y los valores.

En este mismo orden la competencia es considerada como una característica propia de un individuo que está directamente relacionada a un estándar de efectividad o a un desempeño 
superior. Son comportamientos observables en la realidad cotidiana del trabajo y en situaciones de evaluación; son un rasgo de unión entre las características individuales y las cualidades requeridas para el desempeño en una empresa (Alles, 2006).

Teniendo en cuenta lo anterior se puede decir, que la gestión por competencia permite alienar a las personas que integran una organización para el logro de los objetivos estratégicos. Para que sea eficaz se lleva a cabo a través de un modelo sistémico en el cual todos los subsistemas de recursos humanos deben estar incluidos.

\section{Gestión por competencias}

Esta se puede considerar como el proceso que permitirá identificar las competencias que requiere un puesto de trabajo, para que quien lo desarrolle mantenga un rendimiento elevado o superior a la media, "por ello, varios autores coinciden en señalar que estamos en la era de la revolución de los recursos humanos, cuyo desafío es generar un marco claro y estable para potenciar la cualificación y el enfoque de competencia profesional como el paradigma formativo de estos recursos" (Saravia, 2005).

\section{Gestión del talento humano por competen- cias}

Desde una perspectiva general, la globalización, la apertura económica, la competitividad son fenómenos nuevos a los que se tienen que enfrentar las organizaciones. En la medida que la competitividad sea un elemento fundamen- tal en el éxito de toda empresa, los gerentes o líderes harán más esfuerzos para alcanzar altos niveles de productividad y eficiencia.

Los nuevos esquemas gerenciales son reflejo de la forma como la organización piensa y opera, exigiendo entre otros aspectos: un trabajador con el conocimiento para desarrollar y alcanzar los objetivos del negocio; un proceso flexible ante los cambios introducidos en la compañía; una estructura plana, ágil, reducida a la mínima expresión que crea un ambiente de trabajo que contribuya en la consecución de los objetivos organizacionales.

En este sentido Roger hace ver que la competencia organizacional deberá basarse en los nacientes principios de interdependencia, flexibilidad y asociación (Espín \& Torres, 2014).

Entonces, una de las reglas de juego que debe considerarse para competir estratégicamente en este mundo globalizado debe ser el factor humano, tal como lo expresa Jarillo (1990), al indicar que una empresa real está formada por gente, con funciones y capacidades distintas y los resultados de la empresa no son sino la resultante de la interacción de todas esas personas (Bombón, 2016). De este modo, se evidencia que la función de la gestión del talento humano ha adquirido una significativa importancia en la vida de las organizaciones modernas con la aplicación de nuevas técnicas, teorías y principios que den respuestas al comportamiento humano en la misma. Es por ello que Villegas (citado por Ríos, 2013) seña- 
la que hoy las organizaciones evidencian tres grupos de necesidades básicas:

1. La necesidad de una mayor y más efectiva contribución en el logro de las metas.

2. La necesidad de una verdadera excelencia gerencial que sea capaz de autorenovarse dentro de la dinámica propia del funcionamiento organizacional.

3. La necesidad de asimilar cambios drásticos que se suceden en la administración del personal, y de planificar mejores relaciones de trabajo para el futuro.

Lo anterior, exige una nueva forma de gerenciar el talento humano. Según Dolan, Schuler y Valle (1999), el elemento que actualmente distingue con mayor claridad la gerencia estratégica de los recursos humanos es el de su vinculación directa a la estrategia empresarial (Ríos, 2013). Es por ello, que a través del desarrollo humano se logra una cultura superior en la empresa. Es así como la conducción de los recursos humanos a través del enfoque estratégico de competencias promueve con sus resultados la nueva manera de pensar y de desarrollar a la gente, para que se transformen en los principales activos de la organización y sean los mismos quienes aporten con sus competencias al logro de la misión, visión y metas organizacionales.

Concluyendo aquí con lo referente al origen de la gestión por competencias, se presenta a continuación algunas corrientes teóricas que explican las diferentes maneras de abordar la misma.

\section{Corrientes diferenciadoras en la gestión por competencias}

\section{Enfoque Anglosajón}

Se centra en el contenido del puesto de trabajo, así como en su relación con la estrategia global de la organización. Se requiere establecer relaciones causales, lo cual hace a este enfoque muy potente desde el punto de vista metodológico. Para establecer estas relaciones se requiere definir una serie de indicadores observables que actúen como los agentes que causan los rendimientos superiores (variables predictivas o agentes causales).

Según este enfoque, estas relaciones son más difíciles de establecer en puestos que directamente no generan valores o en puestos donde no se conocen los resultados concretos y está orientada hacia las competencias genéricas y universales. Este paradigma considera a las competencias como el lazo que une las conductas individuales con la estrategia de la organización, la cual debe estar sostenida por una cultura adecuada, siendo aquí donde las competencias entran en juego, a través de la gestión estratégica de los Recursos Humanos. Para este enfoque, las competencias reflejan una serie de valores que en muchos casos aglutinan la misión y el plan estratégico, lo que posibilita que aquellas "requeridas" por la organización, puedan ser identificadas a través de un panel de expertos, por lo que en consecuencia se le confiere la utilidad que tienen para cambiar conductas ligadas a los valores estratégicos. Algunos de los autores más representativos de este enfoque son: Boyatzis, Hammel y Prahalad. 


\section{Enfoque Francés}

Se centra más en la persona, y al contrario del enfoque anterior su finalidad es actuar como elemento de auditoría en torno a la capacidad individual del sujeto y el esfuerzo de la organización por mantener su fuerza de trabajo en condiciones óptimas de "empleabilidad".

Considera las competencias como una mezcla indisoluble de conocimientos y experiencias laborales en una organización específica (competencias + experiencias + conocimientos + rasgos de personalidad) /organizaciones específicas, que son las que tienen la capacidad de capacitar a las personas, en función de que estas puedan ejercer de la mejor manera posible sus funciones. Por lo tanto, las experiencias profesionales son la única manera de adquirir competencias que no son reconocidas por un certificado de estudios. Este enfoque enfatiza en la existencia de competencias específicas, lo cual queda reflejado en el uso del concepto autoimagen, como el motor de aprendizaje en las personas, siendo este el que nos proporciona la imagen que tenemos de sí mismos y de por qué nos comportamos de la manera en que lo hacemos, se centra en los procesos de aprendizajes de las personas. Claude Levy Leboyer es uno de los autores más citados como representante de esta corriente (Beltrán \& Urrea, 2013).

Tipos de competencias organizacionales Competencias Transversales

Se refieren a aquellas capacidades que debe exhibir en cada miembro de la entidad. Usual- mente están relacionadas con la Core Competence, es decir, esa competencia central de la que se deriva una posición competitiva. Cuando se menciona que debe ser visible en el desempeño de cada colaborador y directivo se refiere que de ello no se excluye nadie: Directivos, Líderes, Colaboradores. Se sugiere que se priorice y en consecuencia defina la de mayor impacto y se desarrolle.

\section{Competencias Genéricas}

Levy Loboyer especifica que son aquellas que poseen mayor nivel de transferibilidad de unas profesiones a otras. Mazariegos y otros siguen la misma clasificación afirmando que en las competencias genéricas se deben tener en cuenta las capacidades cognoscitivas o aptitudes, los conocimientos que la persona haya adquirido a nivel teórico y a través de las experiencias, las tendencias de su comportamiento o actitudes; sus valores y rasgos de personalidad, así como sus habilidades y destrezas.

Por tanto, en una aproximación a la definición realizada por estos autores, podría afirmarse que las competencias genéricas abarcan capacidades comunes a profesiones diversas o al conjunto de un grupo de profesionales, caracterizándose por poseer un nivel elevado de transferibilidad (Ceinos, 2008).

\section{Competencias Específicas}

Sebastián y Sánchez defienden que los perfiles profesionales exigen una serie de competencias específicas (van-der Hofstadt \& Gras, 2013). Las competencias específicas, son 
aquellas propias de un empleo o conjunto de puesto afines, por lo que no son aplicables a una variedad de empleos, son competencias muy concretas.

Son aquellas capacidades requeridas para que el desempeño en un cargo sea superior al promedio o igual al esperado según la antigüedad del funcionario en el mismo.

Para Spencer y Spencer las competencias pueden clasificarse en:

Competencias de logro a acción: Preocupación por el orden, calidad y precisión, Iniciativa, Búsqueda de información.

Competencias de ayuda y servicio: Entendimiento interpersonal, Orientación al cliente.

Competencias de influencia: Influencia e impacto, Construcción de relaciones, Conciencia Organizacional.

Competencias gerenciales: Desarrollo de personas, Dirección de Personas, Trabajo en equipo, Liderazgo.

Competencias cognoscitivas: Pensamiento Analítico, Razonamiento conceptual, Experiencia técnica, profesional y de dirección.

Competencias de eficacia personal: Autocontrol, Confianza en sí mismo, Comportamiento ante los fracasos, Flexibilidad.

Lo anterior es lo expuesto por un autor nor- teamericano, pero ahora veremos el abordaje de una autora francesa (Levy-Leboyer), quien plantea que las competencias son una serie de comportamientos que ciertas personas poseen más que otras, que las transforman en más eficaces para una situación dada (Moreno, 2014).

Esos comportamientos son observables en la realidad del trabajo y también en situaciones de evaluación. Ellos aplican de manera integral sus aptitudes, sus rasgos de personalidad y sus conocimientos adquiridos. Las competencias representan un rasgo de unión entre las características individuales y las cualidades requeridas para conducir muy bien las misiones profesionales.

La investigadora María Cristina Ceinos Sanz (2008), presenta una lista de competencias universales para los cuadros superiores: Presentación oral, Comunicación oral, Comunicación escrita, Análisis de problemas de la organización, Comprensión de los problemas de la organización, Análisis de problemas fuera de la organización, Comprensión de los problemas fuera de la organización, Planificación y organización, Sensibilidad, Autoridad sobre individuos, Autoridad sobre grupos, Tenacidad, Negociación, Vocación para el análisis, Sentido común, Creatividad, Toma de riesgos, Decisión, Conocimientos técnicos profesionales, Energía, Apertura a otros intereses, Iniciativa, Tolerancia al estrés, Adaptabilidad, Independencia y Motivación (Elorriaga \& Barreto, 2013). 
Para otra autora francesa (Jolis), las competencias son diferentes y se correlacionan entre sí; se dividen en: Competencias teóricas, son aquellas que conectan saberes adquiridos durante la formación con información. Competencias prácticas, son las que traducen la información y conocimientos en acciones operativas. Competencias sociales, destacan la capacidad para relacionarse. Competencias del conocimiento, son las que conjugan la información con saber, coordinar acciones, buscar nuevas soluciones, las tres primeras convergen en la última según Jolis (Márquez, Ojeda \& Romero, 2013).

\section{Diversidad en la Taxonomía de Competencias} La tabla que se presenta a continuación registra las diferentes clasificaciones que hay sobre la tipología de competencias. La diversidad de criterios y términos, podría generar confusión o ambigüedad a la hora de implementar un modelo (Ortiz, Rendon \& Atehourtua, 2014).

Tabla 1. Clasificación de las competencias según distintos autores

\begin{tabular}{|l|l|}
\hline \multicolumn{1}{|c|}{ Autores } & \multicolumn{1}{c|}{ Clasificación } \\
\hline \multirow{4}{*}{ Richard Boyatzi } & 1. Gestión y acción por objetivos \\
\cline { 2 - 2 } & 2. Liderazgo \\
\cline { 2 - 2 } & 3. Gestión de recursos humanos \\
\cline { 2 - 2 } & 4. Dirigir subordinados \\
\cline { 2 - 2 } & 5. Enfocar a otras personas \\
\hline \multirow{4}{*}{ Hay /Mcber } & 1. Competencias principales genéricas \\
\cline { 2 - 2 } & $\begin{array}{l}\text { 2. Competencias genéricas suplementa- } \\
\text { rias y específicas del cliente }\end{array}$ \\
\cline { 2 - 2 } & 3. Competencias emergentes \\
\hline \multirow{2}{*}{$\begin{array}{l}\text { Lyle Spencer y } \\
\text { Singe Spencer }\end{array}$} & 1. Competencias de logro y acción \\
\cline { 2 - 2 } & 2. Competencia de ayuda y servicio \\
\hline
\end{tabular}

\begin{tabular}{|c|c|}
\hline & 3. Competencia de impacto e influencia \\
\hline & 4. Competencias gerenciales \\
\hline & 5. Competencias cognitivas \\
\hline & 6. Competencias de efectividad personal \\
\hline & 7. Otras competencias \\
\hline \multirow{5}{*}{ HayGroup } & 1. Competencias de gestión personal \\
\hline & $\begin{array}{l}\text { 2. Competencias de gestión del equipo } \\
\text { de trabajo }\end{array}$ \\
\hline & 3. Competencias de influencia \\
\hline & 4. Competencia cognitiva \\
\hline & 5. Competencia de logro \\
\hline \multirow{5}{*}{$\begin{array}{l}\text { Alvaro } \\
\text { De Ansorena Cao }\end{array}$} & 1. Metahabilidades \\
\hline & 2. Betahabilidades \\
\hline & 3. Habilidades operativas \\
\hline & 4. Habilidades interpersonales \\
\hline & 5. Habilidades directivas \\
\hline \multirow{2}{*}{ Daniel Goleman } & 1. Competencia personal \\
\hline & 2. Competencia social \\
\hline \multirow{9}{*}{$\begin{array}{l}\text { Jose Luis } \\
\text { Dirube M. }\end{array}$} & 1. Competencias individuales \\
\hline & De acción \\
\hline & De regulación de conducta \\
\hline & De pensamiento \\
\hline & 2. Competencias sociales: \\
\hline & De ayuda \\
\hline & De influencia \\
\hline & De gerencia \\
\hline & De pensamiento \\
\hline \multirow{5}{*}{$\begin{array}{l}\text { Martha Alicia } \\
\text { Alles }\end{array}$} & 1. Competencias cardinales o generales \\
\hline & 2. Competencias específicas \\
\hline & Para nivel ejecutivo \\
\hline & Para nivel gerencial intermedio \\
\hline & Para niveles iniciales \\
\hline \multirow{5}{*}{$\begin{array}{l}\text { Claude } \\
\text { Levy-Leboyer }\end{array}$} & 1. Competencias universales \\
\hline & 2. Supra competencias \\
\hline & 3. Interpersonales \\
\hline & 4. Adaptabilidad \\
\hline & 5. Orientación hacia los resultados \\
\hline
\end{tabular}

Fuente: Ortiz et al. (2014) 


\section{Modelo de competencias}

Un modelo de gestión por competencia permite, desarrollar las prácticas de gestión humana en pro del desarrollo de las persona en relación con las competencias necesarias para alcanzar las estrategias de la organización. Los tres pilares de la implementación del modelo son: Selección, desempeño y desarrollo (Alles, 2011).

El modelo de gestión por competencia nos facilita un adecuado desempeño del rol de cada empleado dentro de su área y se convierte también en una herramienta valiosa para planificar la trayectoria de desarrollo y el mapa de carrera de un empleado dentro de una organización (Ortiz et al., 2014).

Modelo de Iceberg

Este permite entender los componentes de la competencia; en el sentido que es más fácil identificar las competencias relacionadas con las destrezas y conocimiento. Con este modelo los autores (Spencer \& Spencer) representan que las competencias provienen de las características subyacentes de la personalidad, a partir de las cuales se puede predecir el comportamiento de diversas situaciones laborales (Ferreyra, 2014).

Modelo de gestión por competencias de Martha Alles

Dentro de una organización es importante tomar en cuenta la gestión por competencias, ya que gracias a su implementación se pueden obtener óptimos beneficios para la organización. Según la consultora Martha Alles (2006), "para trabajar con un sistema de gestión por competencias, es necesario definir en primer lugar, la visión de la organización: hacia dónde vamos, los objetivos y la misión: que hacemos; y a partir de la misma conducción de la em-

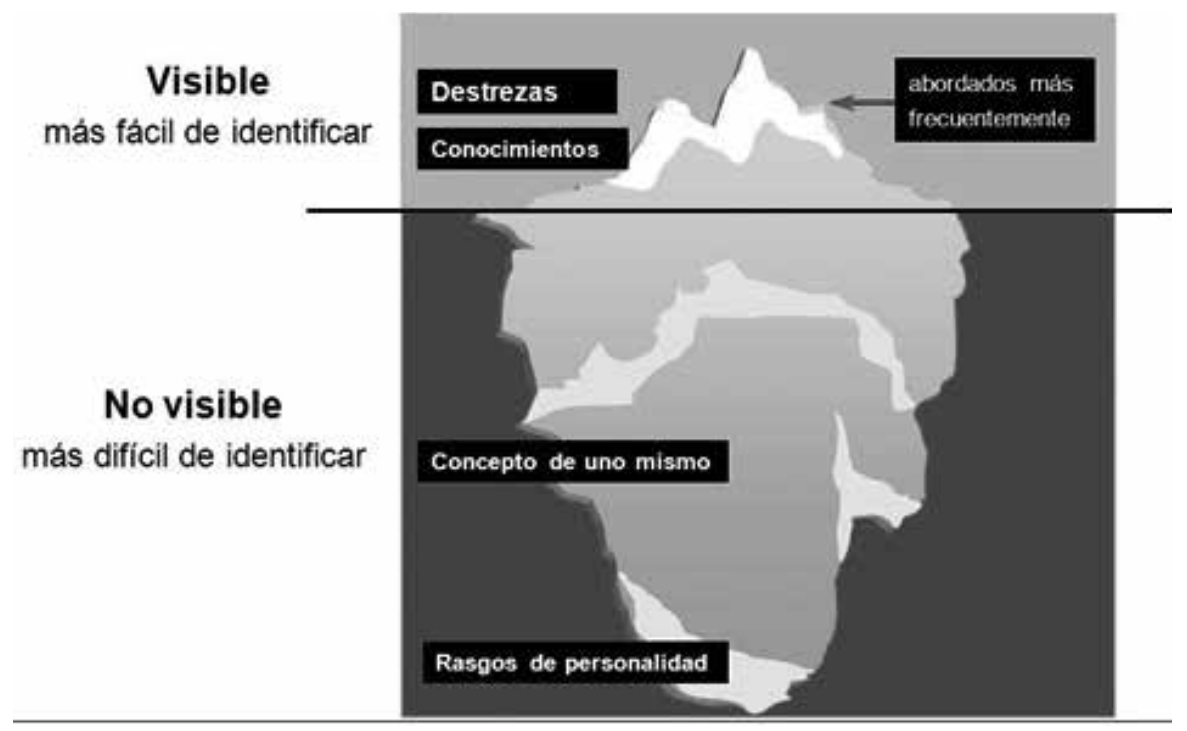

Figura 1. Modelo de Iceberg

Fuente: Spencer y Spencer (Aguilera \& Marchant, 2014) 
presa, con su participación e involucramiento, decidir cómo lo hacemos".

A continuación, se describirán cada uno de los pasos necesarios a seguir para la implementación de un sistema de gestión por competencias. En este caso cabe señalar que diversos autores (Zamora, 2010; Alles, 2006; Saracho, 2005; Gramigna, 2002; Fernández \& Baeza, 2002), presentan similitudes en cuanto al proceso de implantación. La implantación se operacionaliza por medio de los siguientes pasos: definir criterios de desempeño, identificar una muestra, recoger información y definición de las competencias, validar el modelo de competencias, y aplicar el modelo a los subsistemas de recursos humanos (González, Rodríguez, Marimón, Pérez \& López, 2015).

1. Definir criterios de desempeño: consiste básicamente en establecer los criterios de eficiencia superior en desempeño profesional de tarea en estudio. Los criterios recomendados a utilizar son aquellos que proveen información objetiva, es decir, aquellos que se expresan en números, por ejemplo: las ventas, beneficios, patentes, etc.

2. Identificar una muestra: consiste en identificar los grupos modelos para establecer los niveles de cada competencia. Se identifican tres grupos: uno de desempeño superior, otro promedio, y uno mínimo o por debajo del promedio. Es importante mencionar que existen tres factores que influyen en la selección de personas:

I. Los empleados con desempeño superior deben ser los mejores, son aquellos que calificaron alto en todos o en la mayoría de los criterios de desempeño.

II. Es necesario que haya un grupo de control de contraste de empleados con un desempeño totalmente aceptable (promedio); $y$

III. Las muestras deben ser suficientemente grandes para permitir el análisis estadístico.

3. Recoger información: consiste en la aplicación de un método de evaluación, esto implica profundizar previamente, el conocimiento de cada cargo que incluya un análisis descriptivo, además de una visita al lugar de trabajo y así realizar la entrevista, pues es un método recomendable por su efectividad. La que se utiliza es "la entrevista de eventos conductuales", esta se centra en la identificación de los conocimientos, habilidades, actitudes, y soluciones (características) requeridas para que el trabajo sea bien ejecutado.

Los pasos para conducir la entrevista y se logre el propósito son los siguientes.

I. Introducción y explicación: Presentar al entrevistador y explicar el fin de la entrevista.

II. Responsabilidad del puesto: consultar la misión, tareas, responsabilidades, y funciones más relevantes del entrevistado en su puesto.

III. Eventos conductuales: obtener la descripción detallada de los sucesos más importantes que ha experimentado el entrevistado en su trabajo. 
IV. Cierre y recapitulación: agradecimiento por el tiempo y colaboración del entrevistado, recapitulación de la información recabada durante la entrevista.

4. Análisis de información y definición de las competencias: este paso es el más relevante de la intervención y por lo tanto, es el que presenta una mayor complejidad. Consiste en identificar qué características o combinación de características, poseen las personas de desempeño superior. Este proceso es realizado por expertos y se basa en una técnica denominada "Análisis Temático".

Es un método que permite medir empíricamente y comprobar estadísticamente la significación de las diferencias que se detectan en las características demostradas por personas con actuación superior, adecuada en un puesto de trabajo.

5. Validar el modelo de las competencias: existen diferentes maneras de validar este modelo, siendo la más habitual, la realización de una segunda entrevista de eventos conductuales con un nuevo grupo de personas con el fin de comprobar, si las competencias detectadas efectivamente se relacionan con una actuación superior a la tarea.

6. Aplicar el modelo a los subsistemas de recursos humanos: en este último paso es importante resaltar que a las personas se les va a aplicar el modelo en todos los subprogramas de gestión humana, tales como: la selección, capacitación y entrenamiento, desarrollo, evaluación de desempeño, pla- nes de carrera y sucesión y remuneraciones.

\section{Metodología recomendada para implementar} un sistema de gestión por competencias

Para trabajar con un esquema por competencias es necesario "iniciar por el principio" esto es, definir visión, misión y los objetivos y a partir del compromiso de la gerencia decidir cómo se hace (Alles, 2013).

- Definir visión, misión y objetivos.

- Definición de competencias por la máxima dirección de la compañía.

- Prueba de las competencias en un grupo de ejecutivos de la organización.

- Validación de las competencias.

En el diseño de los procesos de recursos humanos por competencias resulta imprescindible que la dirección de la compañía participe antes y durante la instrumentación del sistema de gestión por competencias. Los pasos necesarios son:

- Definir criterios de desempeño.

- Identificar una muestra.

- Recoger información.

- Identificar tareas y los requerimientos en materia de competencias de cada una de ellas; esto implica la definición final de la competencia y su correspondiente apertura en grados.

- Validar el modelo de competencias.

- Aplicar el modelo a los subsistemas de recursos humanos: selección, entrenamiento y capacitación, desarrollo, evaluación de desempeño, planes de sucesión y esquemas de remuneraciones. 
Aplicación de un esquema de competencias por niveles (Alles, 2013)

A continuación, se dan ejemplos de una competencia con sus grados y perfiles por competencias. Se tomaron solo para explicar la idea central de la competencia "trabajo en equipo" con sus cuatro grados ( $A$ el más alto y $D$ el más bajo).

A: Alto o desempeño superior. Según Spencer y Spencer, significa una desviación tipo por encima del promedio del desempeño. Aproximadamente una de cada diez personas alcanza el nivel superior en una situación laboral.

B: Bueno, por sobre el estándar.

C: Mínimo necesario para el puesto, pero dentro del perfil requerido. Por lo general esto significa un nivel "mínimamente aceptable" de trabajo. Es el punto que debe alcanzar un empleado.

D: No se le considera competente para el puesto.

Cómo aplicar cada uno de los distintos procesos de recursos humanos a la gestión por competencia

Selección

Para seleccionar por competencias primero deberán elaborarse los perfiles y las descripciones de puestos por competencias, tendrá competencias derivadas del conocimiento y las competencias de gestión o derivadas de las conductas.

Alles (2011), expone que la base de la selección es la definición del perfil, una correcta división de estos requisitos será clave en las posteriores etapas de la selección, la empresa que trabaja por gestión de competencia debe definir sus competencias para el puesto.

\section{Entrevistas por competencias}

La entrevista es la herramienta por excelencia en la selección de personal (IFEF, 2012). Tiene gran influencia en la decisión de contratación de una persona. Una entrevista de selección por competencias es un diálogo fundamentado en la necesidad de detectar capacidades en el candidato más que los conocimientos necesarios para desempeñar el cargo (Alles, 2006).

\section{Evaluación por competencia}

La evaluación del desempeño por competencias busca determinar si sus empleados están al nivel que ella misma exige al momento de contratar, por ello debe tener en cuenta esas competencias establecidas en el perfil, para tomar decisiones en cuanto a las necesidades de competencias que garanticen el desarrollo de la organización. Esto no significa cambiar a sus ejecutivos y otros colaboradores si el resultado no es el esperado. Simplemente deberá capacitar, entrenar, o mover de puesto a quien lo requiera.

Planes de carrera y planes de sucesión Estos planes deben combinar los requerimientos de conocimientos y habilidades específicas con las competencias conductuales requeridas. Las mismas cambian y evolucionan según la evolución del mapa de puesto. Para los planes de sucesión las competencias deberán 
ser analizadas con relación al individuo y a lo requerido por el puesto al cual se prevé promoverlo en el futuro.

Capacitación y entrenamiento

Para implementar programas de capacitación y entrenamiento por competencias, además de definir las competencias será necesario conocer las del personal. Ello es posible por distintos caminos, a partir de las evaluaciones por competencias o evaluaciones de desempeño.

Desarrollo de los recursos humanos

Si una empresa tiene descripciones de puestos por competencias, planes de carrera con relación a ellos y evalúa el desempeño de su personal por competencias, podrá desarrollar sus recursos humanos con relación a las competencias de la organización, su visión, su misión y los valores.

Evaluación de desempeño

Para evaluar el desempeño por competencias, primero es necesario tener la descripción de puestos por competencias y con base en esto se identifican las competencias transversales y específicas que demanda el cargo que se está evaluando (Alles, 2006).

Evaluación de $360^{\circ}$. Esta es una de las herramientas más completas y novedosas para evaluar el desempeño, ya que evalúa el desempeño de las personas teniendo en cuenta la satisfacción de las necesidades y expectativas, no solo de su jefe sino de todos aquellos que reciben sus servicios, tanto internos como externos. Esta consiste en que un grupo de per- sonas valoren a otra por medio de una serie de ítems o factores predefinidos, estos factores son comportamientos observables en el desarrollo diario de la práctica profesional.

\section{Compensación por competencias}

Se considera el módulo de más difícil implementación. Compensar por competencias significa que la empresa deberá implementar sistemas de remuneración variable donde se consideran para el cálculo, entre otros elementos, las competencias de los colaboradores con relación al puesto y a su desempeño. Es imprescindible evaluar el desempeño por competencias.

\section{Capacidades dinámicas}

Mejorar la competitividad se ha convertido en un objetivo de máxima prioridad para las organizaciones, para hacer frente a los retos de la globalización, por lo que requieren desarrollar capacidades que les permitan hacer frente a estos retos (Uriona, Coelho \& Murcia, 2013). Esta perspectiva se fundamenta en la idea de la consecución de la ventaja competitiva sostenible a través de la posesión por parte de la empresa de un conjunto de recursos únicos, valiosos, inimitables e insustituibles, combinados e integrados para dar lugar a capacidades organizativas distintivas (Rodríguez, Ruiz \& Armario, 2012). Es así como en el ámbito de la economía y de la dirección de empresas se destaca actualmente el papel estratégico que juega el conocimiento en la creación de valor y ventaja competitiva de las organizaciones (Acosta, Longo-Somoza \& Fischer, 2013). 
El mundo actual de las organizaciones está inmerso en un ambiente de competitividad, que a su vez exige a las organizaciones una renovación continua, adaptación y procesos de reorganización de recursos y capacidades al mismo ritmo que evoluciona el mercado.

Las capacidades dinámicas han recibido mucha atención en los campos de la estrategia, la administración y la economía porque ayudan a entender cómo las empresas enfrentan los cambios en el entorno (Carattoli, 2013). Cada organización cuenta con recursos, tales como los recursos financieros, materiales y humanos; la combinación apropiada de estos le permite a la empresa un adecuado funcionamiento y para la administración de esos recursos se necesita que las organizaciones sean dinámicas y se muevan en dirección hacia las necesidades y demandas del mercado. El rendimiento organizativo es el resultado de la correcta alineación de las variables organizativas endógenas con las variables del contexto, o exógenas, estas ideas fueron planteados por Zahra, Sapienza y Davidsson, Wang y Ahmed, (Cruz, Navas, López \& Delgado, 2006). Interpretar el funcionamiento de una organización bajo esta perspectiva nos lleva al concepto de capacidades dinámicas, las cuales se conciben como elementos que generan ventaja competitiva para la organización (Gómez, 2014).

El tema de las capacidades dinámicas analiza las fuentes y métodos de creación de riqueza por las firmas que operan en entornos de rápido cambio tecnológico. La ventaja competitiva de las empresas está sujeta a procesos distintivos, compuestos por la posición específica de los activos en la empresa y la manera cómo han evolucionado o se han heredado (Teece, Pisano \& Shuen, 1997; Acosta et al., 2013; Solano, 2016).

Las capacidades dinámicas buscan la sostenibilidad y competitividad de las organizaciones: "podríamos decir que se ha ocupado de la cuestión fundamental de cómo las empresas desarrollan habilidades y competencias que les permiten competir y obtener ventajas competitivas duraderas" (Carattoli, 2013). Las capacidades dinámicas son la habilidad de la organización para integrar, construir y reconfigurar competencias internas y externas en función de cambios rápidos en el entorno (Acosta et al., 2013; Carattoli, 2013; Teece et al., 1997), entonces las Capacidades Dinámicas se pueden ver como competencias de alto nivel que pueden llegar a fijar habilidades de una organización para integrar, construir y reconfigurar competencias y/o recursos tanto a nivel interno como externo para enfrentar entornos de negocios de alto dinamismo (Elorriaga \& Barreto, 2013).

Las Capacidades Dinámicas pueden ser útiles para tres tipos de actividades: 1) Identificación y evaluación de una oportunidad; 2) Movilización de recursos para aprovechar una oportunidad y capturar valor al hacerlo y 3) Renovarse continuamente (Carattoli, 2013). Esto quiere decir, que las Capacidades Dinámicas son para la organización la capacidad que esta tiene para enfrentar las exigencias del entor- 
no haciendo uso del potencial y dinamismo de los recursos de que dispone, tanto físicos como materiales, permitiendo que se puedan adaptar a los cambios que el entorno exige. "Consideradas hoy capacidades empresariales de primer orden, son rutinas o procesos complejos y de nivel superior" (Vivas-López, 2013).

Por otro lado se encuentra a Eisenhardt y Martin (Henao, López \& Garcés, 2014) quienes indican que las capacidades dinámicas son procesos organizacionales que utilizan recursos. Específicamente los procesos para integrar y reconfigurar, para incluso crear cambios en el mercado (Bravo, Mundet \& y Suñé, 2008). Ellos manifiestan la importancia del mercado en el desarrollo de las capacidades dinámicas

Eisenhardt y Martin, plantean también que las capacidades dinámicas son las rutinas organizacionales y estratégicas por las cuales las firmas logran nuevas configuraciones de recursos mientras el mercado emerge, colisiona, se divide, evoluciona o muere (Carattoli, 2013). Las capacidades dinámicas son consideradas como elementos de naturaleza latente, es decir, que están presentes en el desarrollo de las actividades organizacionales, pueden surgir en momentos de suma necesidad o máxima efervescencia para mejorar los procesos (Carattoli, 2013).

Los planteamientos de Teece están dirigidos hacia la creación de nuevos productos, servicios o procesos, mientras que los postulados de Eisenhardt y Martin se dirigen más a la generación de valor agregado (Cardona, 2013).
Otro gran exponente del tema de Capacidades Dinámicas es Winter quien en compañía de Zollo, quienes las consideran como un patrón aprendido y estable de actividad colectiva a través del cual una empresa sistemáticamente genera y modifica sus rutinas operativas con el propósito de mejorar la eficiencia (Carattoli, 2013).

Winter resalta la incidencia de estas habilidades por su aptitud para modificar las capacidades ordinarias y convirtiéndolas en capacidades difíciles de replicar por otras organizaciones (Rivera \& Figueroa, 2013).

Los autores antes mencionados coinciden en su mayoría en aspectos tales como: el Dinamismo del Entorno, este permite identificar las características del mercado permitiéndole a la empresa adaptarse a los cambios que en este se presentan (Cruz et al., 2006). El Dinamismo del Entorno podría variar por motivos tales como: la Oferta que abarca (tecnología, materia prima, productos o servicios y competidores, entre otros), la Demanda que implica las necesidades del consumidor o el volumen de la misma y por último tenemos el factor macroeconómico el cual está determinado por las políticas gubernamentales, globalización y las crisis de la economía (Cruz et al., 2006).

Otro aspecto es la Capacidad de Adaptación, que se considera como la capacidad que tiene una organización para sobrevivir en su entorno, buscando continuamente oportunidades de crecimiento, adoptando nuevas formas 
de aprendizaje y generación de conocimiento para enfrentar las turbulencias del entorno (Cruz et al., 2006).

Finalmente se puede decir que aunque existes diferentes tipos de capacidades dinámicas, como lo sugiere Teece, quien las clasifica en tres tipos fundamentales: a) La capacidad de percibir oportunidades y amenazas; b) La capacidad de aprovechar las oportunidades; y c) La capacidad para mantener la competitividad a partir de mejorar, combinar, proteger $y$, cuando sea necesario (Carattoli, 2013). Otros autores las clasifican de primer y segundo nivel. Siempre va a depender de cada organización la elección del tipo de capacidades que pueda desarrollar. La elección de desarrollar capacidades dinámicas depende directamente del direccionamiento estratégico de la organización. Además, requiere de la propiedad de activos de difícil imitación (conocimiento). (Teece et al., 2007; Henao, López \& Garcés, 2014).

\section{Referencias}

Acosta, J., Longo-Somoza, M. \& Fischer, A. (2013). Capacidades dinámicas y gestión de conocimiento en nuevas empresas de base tecnológica. Cuadernos de Administración, 35-62.

Aguilera, A. (2010). Gestión humana y estrategia organizacional. Obtenido de http:// www.ascolfa.edu.co/

Aguilera, F. \& Marchant, L. (2014). Competencias (o Habilidades) Gerenciales: Una Herramienta Indispensable para el Desarrollo Organizacional. En L. Marchant,
Actualizaciones para el Desarrollo Organizacional (pp. 62-67). Viña del Mar: Universidad de Viña del Mar.

Alles, M. (2006). Desempeño por competencia. Buenos Aires: Granic.

Alles, M. (2006). Dirección Estratégica de Recursos Humanos. Buenos Aires. En Gestión por Competencias. Buenos Aires: Ediciones Granica S. A.

Alles, M. (2006). Direccionamiento Estrategico. Gestión por competencia. Nueva Edición Martha Alles S.A.

Alles, M. (2011). Modelo de compentecia. Obtenido de http://www.marthaalles. com/modelo-de-competencias.php

Alles, M. (2013). Comportamiento Organizacional: cómo lograr un cambio cultural a través de Gestión por Competencias. Buenos Aires: Ediciones Granica.

Beltrán, N. \& Urrea, D. (2013). Diseño e implementación del modelo de gestión por competencias y evaluación del personal según el modelo, para la empresa. Aportes en Línea. Bogotá: Universidad EAN.

Bombón, G. (2016). La motivación de los trabajadores y su incidencia en la coordinación de las áreas de la línea de producción de la empresa calzado LIWI en el cantón Ambato de la provincia de Tungurahua. Ambato: Universidad Técnica de Ambato.

Bravo, E., Mundet, J. \& \& Suñé, A. (2008). Un nuevo enfoque para el estudio de la teoría de las capacidades dinámicas. Obtenido de Universidad Politécnica de 
Cataluña: https://upcommons.upc.edu/ bitstream/handle/2117/2985/comunicacioncio_parapublicacion2R

Carattoli, M. (2013). Capacidades dinámicas: líneas promisorias y desafíos de investigación. Cuadernos de Administración, 165-204.

Cardona, R. (2013). Estrategia basada en los recursos y capacidades. Criterios de evaluación y el proceso de desarrollo. Forum Doctoral, 113-147.

Castro, E., Miguel, D., Elizabeth, X. \& Sánchez, O. (2013). Plan de desarrollo de personal para el fortalecimiento de competencias laborales de los empleados de la Alcaldía Municipal de Santiago Texacuangos departamento de San Salvador. San Salvador: Universidad de El Salvador.

Ceinos, C. (2008). Diagnóstico de las competencias de los orientadores laborales en el uso de las tecnologías de información y de la comunicación. Santiago de Chile: Universidad Santiago de Compostela.

Chiavenato, I. (2011). Administración de Recursos Humanos: El capital humano de las organizaciones. Ciudad de México: McGraw-Hill.

Chiavenato, I. (2013). Gestión del Talento Humano. McGraw-Hill.

Cruz, J., Navas, J., López, P. \& Delgado, M. (2006). Conceptos e implicaciones de las capacidades dinámicas desde un enfoque de dirección del conocimiento. Madrid: Universidad Complutense de Madrid.

Dessler, G. (2009). Administración de Recursos Humanos. México: Pearson.
Dolan, S., Schuler, R. \& Valle, R. (1999). La gestión de los recursos humanos (human resource management). Madrid: McGraw-Hill.

Elorriaga, D. L. \& Barreto, A. (2013). La problemática de la inducción en los puestos operativos en las mipymes de alojamiento turístico de la ciudad autónoma de Buenos Aires (2011-2013). Ad-Gnosis, 2(2), 23-48.

Espín, J. \& Torres, N. (2014). Análisis del clima organizacional y su impacto en el desempeño laboral del personal administrativo y operativo del Servicio Nacional de Aduana del Ecuador Distrito Quito para el periodo 2006-2011. Quito: Universidad Politénica Salesiana.

Fernandez, I. \& Baeza, R. (2002). Aplicación del modelo de competencias: experiencias en algunas empresas chilenas. Obtenido de PROSEL Consultores: http://www. gestiopolis.com/dirgp/rec/gescomp. htm

Ferreyra, R. (2014). Recursos humanos. Gestión por competencias. Obtenido de Repositorio virtual de la Universidad de Belgrano: http://184.168.109.199:8080/ jspui / bitstream/123456789/ 3579/1/4026\%20-\%20recursos\%20humanos\%20-\%20ferreyra.pdf

Gelabert, M. (2014). Gestión de personas $6 \underline{a}$ ed.: Manual para la gestión del capital humano en las organizaciones. Madrid: Esic Editorial. 
Gómez, N. (2014). ¿Es la gestión del talento humano un factor de competitividad en las Pymes en la ciudad de Pasto? Revista UNIMAR, 29-32.

González, M. (2012). ¿Cómo implementar el modelo de gestión por competencias para incrementar la productividad en las organizaciones? Bogotá: Universidad de la Sabana.

González, M., Rodríguez, A., Marimón, R., Pérez, M. \& López, Y. (2015). La gestión por competencias para el desempeño exitoso, análisis desde el sector empresarial cubano. Avances, 64-75.

González, V. (2002). ¿Qué significa ser un profesional competente? Reflexiones desde una perspectiva psicológica. Revista Cubana de Educación Superior, 45-53.

Gramigna, M. (2002). Gestión por competencias: una opción para hacer a las empresas más competitivas. Obtenido de Belo Horizonte, MRG Consultoria e Treinamento Empresarial: http://www.gestiopolis.com/dirgp/rec/gescomp.htm

Henao, E., López, M. \& Garcés, R. (2014). Medición de capacidades de investigación e innovación en instituciones de educación superior: una mirada desde el enfoque de las capacidades dinámicas. Entramado, 252-271.

IFEF (2012). Manual sobre cómo afrontar una entrevista de selección por competencias. Cádiz: Instituto de Fomento, Empleo y Formación.

Jarillo, J. (1990). Dirección estratégica. Madrid: McGraw-Hill.
Márquez, W., Ojeda, Y. \& Romero, A. (2013). Propuesta de un perfil de competencias gerenciales para la dirección de Deportes de la Universidad de Oriente. Cumaná: Universidad de Oriente.

McClelland, D. (1973). Testing for competence rather than for "intelligence". American Psychologist, 1-14.

Moreno, C. (2014). Las competencias laborales como eje fundamental de la profesionalización de los servidores públicos en iberoamércia. Obtenido de Centro Latinoaméricano de Administración para el Desarrollo: http://siare.clad.org/ fulltext/0078302.pdf

Moreno, L. (2012). Dirección y gestión de recursos humanos. 7a ed. Madrid: Ediciones Díaz de Santos.

Muñoz, S. \& Vargas, M. (2016). Evaluación de las competencias del profesional contable egresado de la Universidad de Antioquia para desempeñarse en el sector financiero de Medellín. Medellín: UdeA. Ortiz, J., Rendon, M. \& Atehourtua, J. (2014). Score de compentencia. Bogotá: McGraw-Hill Interamericana S.A.

Pardo, C. E. \& Porras, J. A. (2011). La gestión del talento humano ante el desafío de organizaciones competitivas. Gestión y Sociedad, 04, 13.

Parker, G., McAdams, J. L. \& Zielinski, D. (2013). Cómo recompensar eficazmente a un equipo de trabajo: 27 ejemplos actuales de planes de compensación y reconocimiento de equipos en compañías líderes. Madrid: Ediciones Díaz de Santos. 
Ríos, J. (2013). Responsabilidad social y gestión del conocimiento como estrategias de gestión humana. Estudios Gerenciales, 110-117.

Rivera, H. A. \& Figueroa, L. S. (2013). Capacidades Dinámicas: Una Fuente de Ventaja Competitiva. Criterio Libre, 06.

Rodríguez, M. \& Sabastián, D. (2014). Diseño de un modelo de evaluación de la gestión de recursos humanos en la empresa Coaching Head Hunting International. Quito: Pontificia Universidad Católica de Ecuador.

Rodríguez, M., Ruiz, C. \& Armario, E. (2012). Las empresas Born Global: un enfoque de capacidades dinámicas. Revista Internacional de la Pequeña y Mediana Empresa, 49-67.

Ruiz, C. \& Rodríguez, W. (2012). Diseño de un programa de evaluación por competencias en Hilanderías Bogotá S.A. Bogotá: Universidad de la Sabana.

Saracho, J. (2005). Un modelo general de gestión por competencias. Santiago: RIL Editores.

Saravia, M. (2005). Recursos humanos en el siglo XXI: Competencias. 1a ed. Bolivia: Editorial MASG.

Solano, A. (2016). Influencia de las capacidades dinámicas en la empresa en el desempeño exportador: un estudio empírio en México. Cantabria: Universidad de Cantabria.

Teece, D. (2007). Explicating Dynamic Capabilities: de nature and microfoundations of (sustainable enterprice performar- se). Strategic Management Journal, 28, 1319-1350.

Teece, D. J., Pisano, G. \& Shuen, A. (1997). Dynamic capabilities and strategic management. Strategic Management Journal, 18(7), 509-533.

Torres, E. (2015). Análisis y diseño de un plan de carrera para el área de ventas. Caso: Pasteurizadora Quito S.A. Quito: Universidad Andina Simón Bolívar.

Trujillo, N. (2014). Selección efectiva de personal basada en competencias. Revista do Serviço Público, 99-120.

Uriona, M., Coelho, L. \& Murcia, C. (2013). Capacidades dinámicas de las Pymes en el nuevo entorno de crisis económica. Economía Industrial, 23-34.

Valencia, J. C., Gutiérrez, S. \& Rubio, M. (2013). El rol del capital humano en la generación de valor: Variables determinantes. Revista Ciencias Estrategicas, 31-48.

van-der Hofstadt, C. \& Gras, J. (2013). Competencias y habilidades profesionales para universitarios. Madrid: Ediciones Díaz de Santos.

Vivas-López, S. (2013). Implicaciones de las capacidades dinámicas para la competitividad y la innovación en el siglo XXI. Cuadernos de Administración, 119-140.

Zamora, R. (2010). La gestión por competencias. Obtenido de http://www.traininggames.com/es/base_conceptual/articulos.htm 
